\title{
Efecto del entrenamiento de músculos abdominales sobre la función respiratoria en adolescentes sanos. Estudio piloto
}

\author{
IVÁN RODRÍGUEZ N.***,***, MARCOS ALARCÓN S.****, CAROLINA GUTIÉRREZ G.****, \\ PAOLA HERMOSILLA R.****, TANIA CONTRERAS G.* y CLAUDIO BÁEZ R.*
}

Effect of abdominal muscle training on respiratory function in healthy adolescents. A preliminar study

The respiratory muscle training has been used to improve muscle strength and optimize the mechanism of cough. The aim of this study is to evaluate the effect of abdominal muscle training on respiratory muscle strength (MIP, MEP), peak expiratory flow (PEF) and peak cough flow (PCF) in healthy adolescents. The study design was quasi-experimental, variables of respiratory function were assessed before and after the muscle training protocol in a sample of sedentary healthy adolescents without gender restriction. The sample size calculated was 15 subjects. The training protocol consisted in 8 weeks of training divided into two stages (basic and advanced training plan) twice a week. Descriptive statistics were performed, tests for normality (Shapiro Wilk), U Mann Whitney test, Pearson coefficient and $t$-student test were used. Results are expressed as mean and its standard deviation. It was considered significant a p value $<0,05$. Seventeen subjects ( 8 males and 9 females) entered to the study, all subjects performed the protocol and completed it without incidents. After completing the training protocol the sit-up test performance increased $21.7 \%(\mathrm{p}=0.0001)$, the MIP increase $16,5 \mathrm{~cm} \mathrm{H}_{2} \mathrm{O}$ $(17.1 \%)(p=0.006)$, MEP increased $34.9 \mathrm{~cm} \mathrm{H}_{2} \mathrm{O}(50,2 \%)(p=0.0001)$. Moreover, PEF increased $35.3 \mathrm{~L} / \mathrm{min}(8.3 \%)(p=0.003)$ and $P C F$ increased $36.6 \mathrm{~L} / \mathrm{min}(9,1 \%)$. There was no correlation between sit-up test performance and lung function variables. PEF showed only a moderate correlation with PCF $(r=0.6 ; p=0.007)$ and MEP $(r=0.59 ; p=0.01)$. We conclude that, in this sample, increases in respiratory muscle strength, $P E F$ and $P C F$ were observed after abdominal muscle training. No association between abdominal muscle strength and respiratory function variables was found before initiating the training protocol.

Key words: Respiratory muscle training, peak expiratory flow, respiratory muscles, abdominal muscles, cough.

\section{Resumen}

El entrenamiento muscular respiratorio ha sido utilizado para mejorar la fuerza de los músculos y optimizar el mecanismo de la tos. El objetivo de este estudio es evaluar el efecto del entrenamiento de músculos abdominales sobre la fuerza de los músculos respiratorios (Pimax, Pemax), flujo espiratorio máximo (FEM) y flujo máximo de tos (FMT) en adolescentes sanos. Este estudio es cuasi experimental, donde se evaluaron las variables de función respiratoria antes y después del protocolo de entrenamiento. La muestra estuvo integrada por adolescentes sanos sedentarios sin restricción de sexo. La muestra mínima estimada fue de 15 sujetos. El protocolo de entrenamiento consistió en 8 semanas de entrenamiento dividido en 2 etapas (plan básico y avanzado de ejercicios) dos veces por semana. Se realizó estadística descriptiva, pruebas de normalidad (Shapiro Wilk); se utilizó test

\footnotetext{
* Docente Escuela de Kinesiología, Facultad de Ciencias de la Salud, Universidad San Sebastián. Concepción. Chile.

** Docente Escuela de Kinesiología, Facultad de Salud, Universidad Santo Tomás, Concepción, Chile.

*** Kinesiólogo, Servicio de Pediatría. Hospital Dr. Guillermo Grant Benavente. Concepción. Chile.

**** Interno/a Kinesiología. Universidad San Sebastián Concepción. Chile.
} 
U de Mann Whitney, coeficiente de correlación de Pearson y test $t$-student para muestras pareadas. Los resultados se expresan en promedios y desviación estándar. Se consideró significativo un valor de $p<0,05$. Ingresaron al estudio 17 sujetos (8 hombres y 9 mujeres), todos los sujetos realizaron el protocolo completo. Posterior al protocolo los sujetos incrementaron en 21,7\% el rendimiento del sit-up test $(p=0,0001)$; la Pimax aumentó 16,5 $\mathrm{cm} \mathrm{H} \mathrm{H}_{2} \mathrm{O}(+17,1 \%)(p=0.006)$, la Pemax aumentó 34,9 $\mathrm{cm} \mathrm{H}_{2} \mathrm{O}(+50,2 \%)(p=0,0001)$. Por otra parte, el FEM aumentó 35,3 L/min $(+8,3 \%)(p=0,003)$ y el FMT aumentó 36,6 L/min (+9,1\%). La fuerza de músculos abdominales no muestra correlación con las variables de función respiratoria. Sólo FEM muestra correlación moderada con el FMT ( $r=0,6$; $p=0,007)$ y Pemax $(r=0,59 ; p=0,01)$. Se concluye que, en la muestra estudiada, se observan incrementos en la fuerza muscular respiratoria, FEM y FMT luego de un protocolo de entrenamiento de músculos abdominales. No se observó asociación entre la fuerza muscular abdominal y las variables de función respiratoria antes de iniciado el protocolo.

Palabras clave: Entrenamiento muscular respiratorio, flujo espiratorio máximo, músculos respiratorios, músculos abdominales, tos.

\section{Introducción}

El reflejo de la tos constituye un importante mecanismo de defensa innato del sistema respiratorio puesto que permite la eliminación de agentes extraños y esputo bronquial desde la vía aérea. Su eficacia depende de la capacidad funcional de los grupos musculares inspiratorios y espiratorios, así como también, de las características anátomo-funcionales de la caja torácica ${ }^{1}$.

Existen diversas patologías que cursan con deterioro del mecanismo de la tos. En este contexto, se ha observado en pacientes con enfermedades neuromusculares (ENM) un compromiso severo de la función de la tos, demostrado a través de caídas significativas del flujo máximo de tos (FMT), presión espiratoria (Pemax) e inspiratoria máxima (Pimax). Esto constituye un factor de riesgo importante para la generación de infecciones respiratorias, contribuyendo a incrementar el deterioro en la calidad de vida y pronóstico de este grupo de pacientes ${ }^{2,3}$. Por otra parte, se ha descrito recientemente que los pacientes con fibrosis quística poseen debilidad de los músculos respiratorios y experimentan caídas en la fuerza muscular inspiratoria una vez que son colonizados por Pseudomonas aeruginosa, fenómeno que se acentúa con las infecciones respiratorias recurrentes a lo largo del curso clínico de la enfermedad, constituyendo un factor de riesgo potencial para el deterioro, a largo plazo, del mecanismo de la tos ${ }^{4-6}$.

Diversas estrategias terapéuticas han sido desarrolladas para optimizar el mecanismo de la tos y mitigar las repercusiones de su deterioro. En este ámbito, las técnicas de asistencia manual y el empleo de dispositivos de tos asistida han sido los métodos más efectivos para lograr una adecuada depuración o clearance bronquial en pacientes con debilidad muscular respiratoria e hipersecreción bronquial en estado estable ${ }^{7,8}$. Adicionalmente, el entrenamiento de los músculos respiratorios ha sido empleado para mejorar la función de los músculos inspiratorios y espiratorios con resultados exitosos tanto en sujetos sanos como en pacientes con enfermedades respiratorias crónicas (ERC) $)^{9-11}$. Sin embargo, sus efectos sobre la capacidad generadora de flujos espiratorios forzados, aun son materia de estudio; pese a aquello, el EMR se encuentra actualmente recomendado en las guías nacionales de rehabilitación para niños con enfermedades respiratorias crónicas ${ }^{12}$.

Considerando los discrepantes resultados respecto al beneficio del entrenamiento muscular respiratorio en pacientes con ERC, otras estrategias de intervención han sido evaluadas, como la electro-estimulación de músculos abdominales; lo cual ha mostrado efectos positivos en pacientes con lesión medular alta ${ }^{13,14}$. Resultados que motivan el desarrollo y profundización de esta línea de investigación.

El efecto de un programa de entrenamiento abdominal activo sobre la fuerza de los músculos respiratorios y flujos espiratorios forzados no ha sido reportado, razón por la cual, se hace necesario conocer el comportamiento de estas variables posterior a un programa de entrenamiento localizado de fuerza abdominal, en una muestra de adolescentes sanos, antes de iniciar el diseño de un estudio clínico en pacientes con ERC.

Por lo precedentemente planteado, el objetivo primario de este estudio es evaluar el efecto de un protocolo de fortalecimiento de músculos abdominales sobre la función muscular respiratoria (Pimax, Pemax), flujo espiratorio máximo (FEM) y flujo máximo de tos (FMT); el objetivo secundario de este estudio es determinar la correlación entre las variables de función respiratoria con el nivel de fuerza de los músculos abdominales, 
antes de iniciar el protocolo de entrenamiento, en una muestra de adolescentes sanos sedentarios.

\section{Sujetos y Métodos}

\section{Diseño}

Estudio cuasi experimental, donde se evaluaron las variables de función respiratoria y fuerza de músculos abdominales antes y después de la ejecución del protocolo de entrenamiento.

\section{Muestra \\ A través de un muestreo no probabilístico por conveniencia se seleccionaron 17 adolescentes voluntarios sanos, sin restricción de sexo, estu- diantes del colegio del Amanecer de Talcahuano, de entre 16 y 17 años de edad. Los criterios de inclusión fueron: jóvenes adolescentes sanos sedentarios (que realicen actividad física con una frecuencia menor a dos veces por semana) y que no hayan sido sometidos a entrenamientos de fuerza de músculos abdominales en el último mes. Como criterios de exclusión se conside- ró: cualquier patología cardiorrespiratoria $\mathrm{y} / \mathrm{o}$ músculo-esquelética que impidiera la realización del protocolo, aquellos sujetos que fuman y aquellos que se encuentren consumiendo algún medicamento que modifique los flujos forzados del sistema respiratorio}

\section{Tamaño de muestra}

Considerando una potencia estadística del $85 \%$, riesgo de error alfa $5 \%$, una varianza 25 , una diferencia mínima esperada de la cantidad de abdominales realizado en $30 \mathrm{~s}$, antes y después del entrenamiento de 5; el tamaño muestral estimado fue de 15 sujetos. Los datos empleados para el cálculo fueron extraídos de los resultados de esta serie puesto que son escasos los reportes previos que proporcionen datos confiables para una estimación adecuada del tamaño de muestra. Para el cálculo se utilizó el programa para análisis epidemiológico de datos tabulados EPIDAT 3.1 .

\section{Variables}

Antes de iniciar el protocolo, en una planilla ad-hoc, se registraron las variables biodemográficas (estatura, peso corporal, edad y sexo) y las variables de función respiratoria (Pimax, Pemax, FEM y FMT). Para la medición del peso y talla se utilizó una cinta métrica y balanza análoga cuyo registro se expresa en kilogramos $(\mathrm{kg})$ y centímetros $(\mathrm{cm})$ respectivamente.

Tanto el FEM como el FMT, se midieron con un flujómetro (Mini-Wright ${ }^{\circledR}$; Clement Clarke International, Essex, England). La medición del FEM se llevó a cabo con el sujeto en posición de pié, utilizando una pinza nasal donde debe soplar con la mayor fuerza posible desde capacidad pulmonar total. La prueba se realizó un mínimo de 3 ocasiones y máximo de 8 ; se registró el valor más alto reproducible en 3 intentos con una diferencia no mayor al $10 \%$ entre cada uno de ellos ${ }^{15}$. El FMT se midió con el sujeto sentado indicándole que inspirase lo más profundo posible y que realizara un esfuerzo de tos máximo a través del flujómetro. Los resultados se expresaron en litros/ minuto ${ }^{16}$.

La fuerza de los músculos respiratorios se evaluó a través de la presión inspiratoria máxima (Pimax), medida a través de un esfuerzo inspiratorio máximo mantenido por al menos un segundo desde volumen residual; y con la presión espiratoria máxima (Pemax) medida a través de un esfuerzo espiratorio máximo mantenido por al menos un segundo desde capacidad pulmonar total. Como instrumento se utilizó un manómetro aneroide NS120-TRS calibrado en centímetros de agua (desde 0 a -120 y desde $0 \mathrm{a}+120 \mathrm{cmH}_{2} \mathrm{O}$ ). Los valores obtenidos de Pimax y Pemax se expresan en valores absolutos $\left(\mathrm{cmH}_{2} \mathrm{O}\right)^{17}$.

La fuerza de los músculos abdominales se evaluó a través del Sit-Up test, el cual, corresponde a una prueba funcional que ha sido ampliamente utilizada en pacientes con enfermedades respiratorias crónicas ${ }^{18}$. En la posición inicial el sujeto se encuntra tendido en decúbito supino, las rodillas en flexión de 90 grados con la planta de los pies tocando el suelo y las manos a un costado de la cabeza. Un ayudante mantiene los pies fijos en la colchoneta. En este momento, el examinador indica el comienzo de la prueba que consiste en efectuar el número máximo de ciclos de flexiónextensión de tronco durante $30 \mathrm{~s}$ (Figura 1c).

\section{Protocolo de entrenamiento}

Este protocolo consistió en 8 semanas de entrenamiento de fuerza abdominal que fue dividido en dos etapas, una etapa inicial donde se desarrolló un plan básico de ejercicios y una etapa final donde se desarrolló un plan avanzado de ejercicios; cada etapa tuvo una duración de 4 semanas, se realizaron 2 sesiones por semana y cada sesión de ejercicios tuvo una duración de 45 minutos donde se desarrolló la totalidad de los ejercicios de cada plan. El plan básico estuvo conformado por los siguientes ejercicios: Crunch, cross curl-up, sit-up; foot lateral crunch; elevación de piernas, apoyo lateral estático, puente con elevación de piernas, elevación de rodillas 


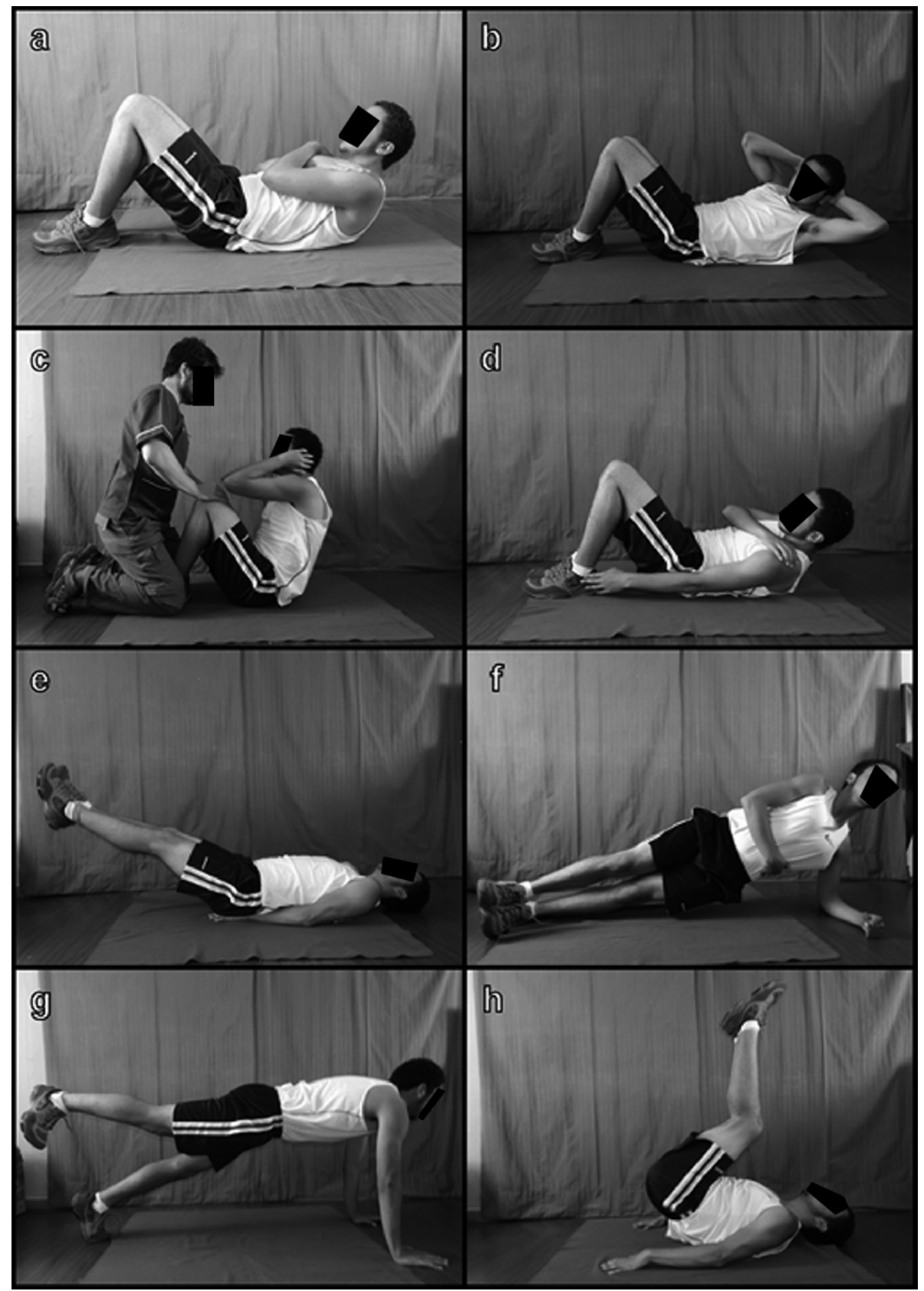

Figura 1. Plan básico de ejercicios. a: Crunch; b: Cross curl-up; c: Sit-up; d: Foot lateral crunch; e: Elevación de piernas; f: Apoyo lateral estático; g: Puente con elevación de piernas; h: Elevación de rodillas tumbado.

tumbado. Por otra parte, el plan avanzado conformado por: Crunch con cadera en $130^{\circ}$, crunch con estiramiento, crunch con piernas separadas, crunch con los pies en alto, escarabajo, lagartija con crunch, apoyo lateral dinámico, elevación de pierna en cuatro tiempos. Cada uno de los ejercicios fue realizado durante un tiempo máximo de un minuto en cada sesión de entrenamiento. El volumen de ejercicio alcanzado fue de entre 200 a 300 repeticiones por sesión. En las Figuras
1 y 2 se muestra cada uno de los ejercicios realizados en el plan básico y avanzado de ejercicios respectivamente.

Todos los sujetos realizaron calentamiento y vuelta a la calma antes y después del protocolo de fortalecimiento.

\section{Consideraciones éticas}

Se contó con la autorización del establecimiento educacional para la realización de este estudio. 


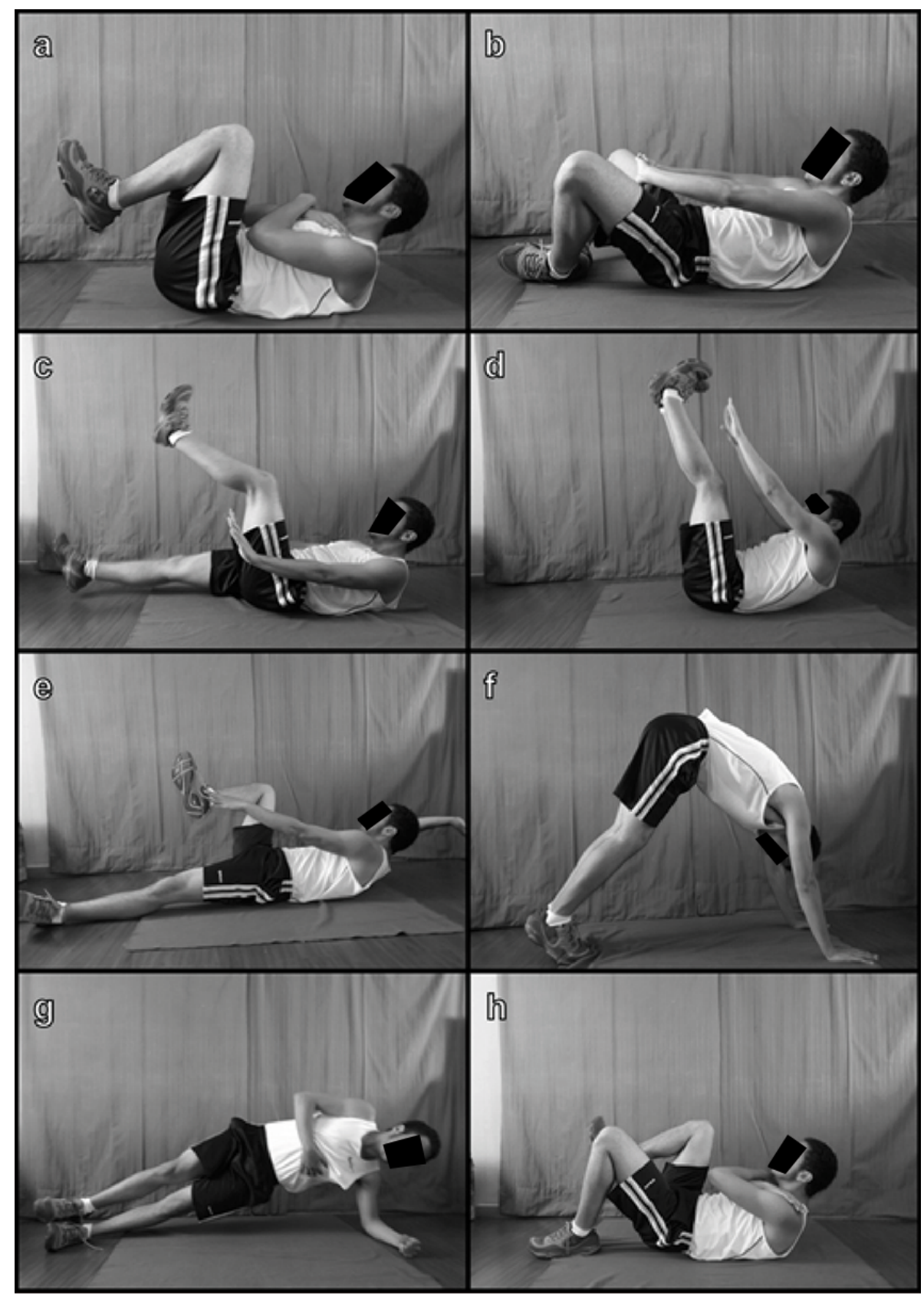

Figura 2. Plan avanzado de ejercicios. a: Crunch con cadera en $130^{\circ}$; b: Crunch con estiramiento; c: Crunch con piernas separadas; d: Crunch con los pies en alto; e: Escarabajo; f: Lagartija con crunch; g: Apoyo lateral dinámico; h: Elevación de pierna en cuatro tiempos.

Adicionalmente todos los padres firmaron un consentimiento informado y los adolescentes enrolados en el estudio firmaron su asentimiento informado.

\section{Análisis estadístico}

Se realizó estadística descriptiva para mostrar las características biodemográficas de la muestra, las cuales son expresadas en promedio y desviación estándar. Se realizaron pruebas de normali- dad (Shapiro Wilk) para evaluar la distribución de las variables; se utilizó análisis de regresión lineal simple (coeficiente de correlación de Pearson) para evaluar la correlación entre las variables cuantitativas. A través de la prueba t-student para muestras pareadas se realizó la comparación de medias antes y después del entrenamiento y a través de la prueba no paramétrica U-Mann Whitney se evaluó la significancia de las diferencias entre las variables según sexo. 
Para el análisis estadístico se utilizó el software SPSS 11,5. Se consideró significativo un valor de $\mathrm{p}<0,05$.

\section{Resultados}

\section{Características de la muestra}

$\mathrm{Al}$ estudio ingresaron 17 sujetos (8 hombres y 9 mujeres), se registraron las características antropométricas, así como también, las variables de fuerza muscular abdominal y de función respiratoria antes de iniciar el protocolo de entrenamiento. Se observa que las mujeres presentan

Tabla 1. Características generales de los adolescentes estudiados

\begin{tabular}{|lc|}
\hline Variables & \\
Sexo $(\mathrm{H} / \mathrm{M})$ & $8 / 9$ \\
Edad (años) & $16,2 \pm 0,4^{*}$ \\
Talla $(\mathrm{cm})$ & $161,4 \pm 8,0$ \\
Peso $(\mathrm{kg})$ & $56,6 \pm 10,0$ \\
\hline
\end{tabular}

H: Hombre; M: Mujer. *Promedio \pm 1 DS. menores niveles de FEM y FMT que alcanzan la significancia estadística. En la Tabla 1 se muestran las características biodemográficas de la muestra y en la Tabla 2 se muestran las variables de función respiratoria y fuerza de músculos abdominales antes de iniciar el protocolo distribuido según sexo.

Efecto del entrenamiento de músculos abdominales sobre las variables de función respiratoria.

Todos los sujetos realizaron el protocolo y lo finalizaron sin incidentes, no hubo pérdidas por falta de adherencia o problemas de salud de alguno de los integrantes de la muestra. Posterior al programa de ejercicios los sujetos incrementaron en $5,1(21,7 \%)$ la cantidad de abdominales ejecutados en $30 \mathrm{~s}(\mathrm{p}=0,0001)$ (Figura 3$)$.

Respecto a la fuerza de los músculos respiratorios la Pimax aumentó $16,5 \mathrm{~cm} \mathrm{H}_{2} \mathrm{O}(17,1 \%)$ $(\mathrm{p}=0,006)$, la Pemax aumentó 34,9 $\mathrm{cm} \mathrm{H}_{2} \mathrm{O}$ $(50,2 \%)(p=0,0001)$. Por otra parte, el FEM aumentó $35,3 \mathrm{~L} / \mathrm{min}(8,3 \%)(\mathrm{p}=0,003)$ y el FMT aumentó $36,6 \mathrm{~L} / \mathrm{min}(9,1 \%)(\mathrm{p}=0,015)$. Ningún sujeto mostró caídas en alguna de las variables estudiadas posterior al protocolo. En la Tabla 2 se muestran los resultados de las variables medidas antes y después del entrenamiento.

Tabla 2. Variables de función respiratoria y fuerza de músculos abdominales pre entrenamiento de músculos abdominales

\begin{tabular}{|c|c|c|c|}
\hline Variables & Hombres $(n=8)$ & Mujeres $(\mathrm{n}=9)$ & $\mathbf{p}$ \\
\hline $\operatorname{Pimax}\left(\mathrm{cm} \mathrm{H}_{2} \mathrm{O}\right)$ & $99,7 \pm 13,1$ & $93,3 \pm 21,7$ & 0,355 \\
\hline Pemax $\left(\mathrm{cm} \mathrm{H}_{2} \mathrm{O}\right)$ & $77,0 \pm 15,0$ & $62,6 \pm 14,1$ & 0,064 \\
\hline FEM (L/min) & $471,2 \pm 79,7$ & $377,7 \pm 84,9$ & 0,048 \\
\hline FMT (L/min) & $443,7 \pm 64,5$ & $363,3 \pm 72,2$ & 0,033 \\
\hline Sit-up test $\left(\mathrm{n}^{\circ} \mathrm{rep} / 30 \mathrm{~s}\right)^{*}$ & $25,6 \pm 4,3$ & $21,6 \pm 3,8$ & 0,052 \\
\hline
\end{tabular}

Pimax: presión inspiratoria máxima; Pemax: presión espiratoria máxima; FEM: flujo espiratorio máximo; FMT: flujo máximo de tos; *Sit-up test: cantidad de repeticiones efectuadas en 30 segundos. Las cifras representan el promedio \pm 1 DS de las variables.

Tabla 3. Variables de función respiratoria antes y después de aplicar el protocolo de entrenamiento de músculos abdominales

\begin{tabular}{|lccc|}
\hline Variables & Pre $(\mathbf{n}=\mathbf{1 7})$ & Post $(\mathbf{n}=\mathbf{1 7})$ & p \\
Pimax $\left(\mathrm{cm} \mathrm{H}_{2} \mathrm{O}\right)$ & $96,3 \pm 17,9$ & $112,8 \pm 18,0$ & 0,006 \\
Pemax $\left(\mathrm{cm} \mathrm{H} \mathrm{H}_{2} \mathrm{O}\right)$ & $69,4 \pm 15,9$ & $104,3 \pm 14,3$ & 0,0001 \\
FEM (L/min) & $421,7 \pm 93,3$ & $457,0 \pm 78,6$ & 0,003 \\
FMT (L/min) & $401,2 \pm 78,4$ & $437,6 \pm 79,3$ & 0,015 \\
\hline
\end{tabular}

Pimax: presión inspiratoria máxima; Pemax: presión espiratoria máxima; FEM: flujo espiratorio máximo; FMT: flujo máximo de tos. Las cifras representan el promedio \pm 1 DS de las variables. 


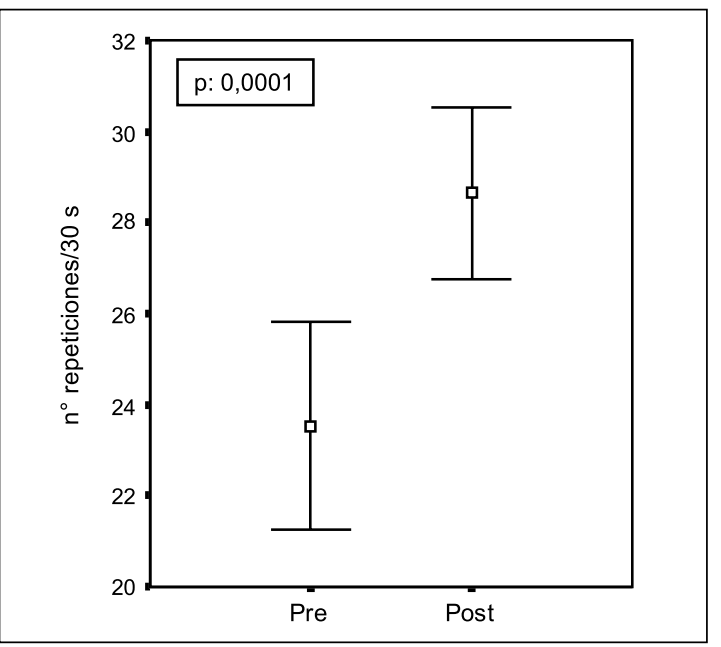

Figura 3. Sit-up test antes y después del protocolo de entrenamiento. Se representa la media $(\square) \pm 1$ DS.

\section{Correlación entre FMA y variables de función respiratoria}

La FMA antes de iniciar el protocolo muestra una correlación débil (no significativa) con las variables de función respiratoria (Pimax, Pemax, FEM, FMT). Sólo el FMT muestra una correlación moderada con el FEM $(r=0,6 ; p=0,007)$ y Pemax $(r=0,59 ; p=0,01)$.(Figuras 4 y 5 respectivamente).

\section{Discusión}

En este estudio piloto se pretendió evaluar el comportamiento de las variables de función respiratoria luego de la ejecución de un protocolo de entrenamiento de fuerza de músculos abdominales en una muestra de adolescentes sedentarios.
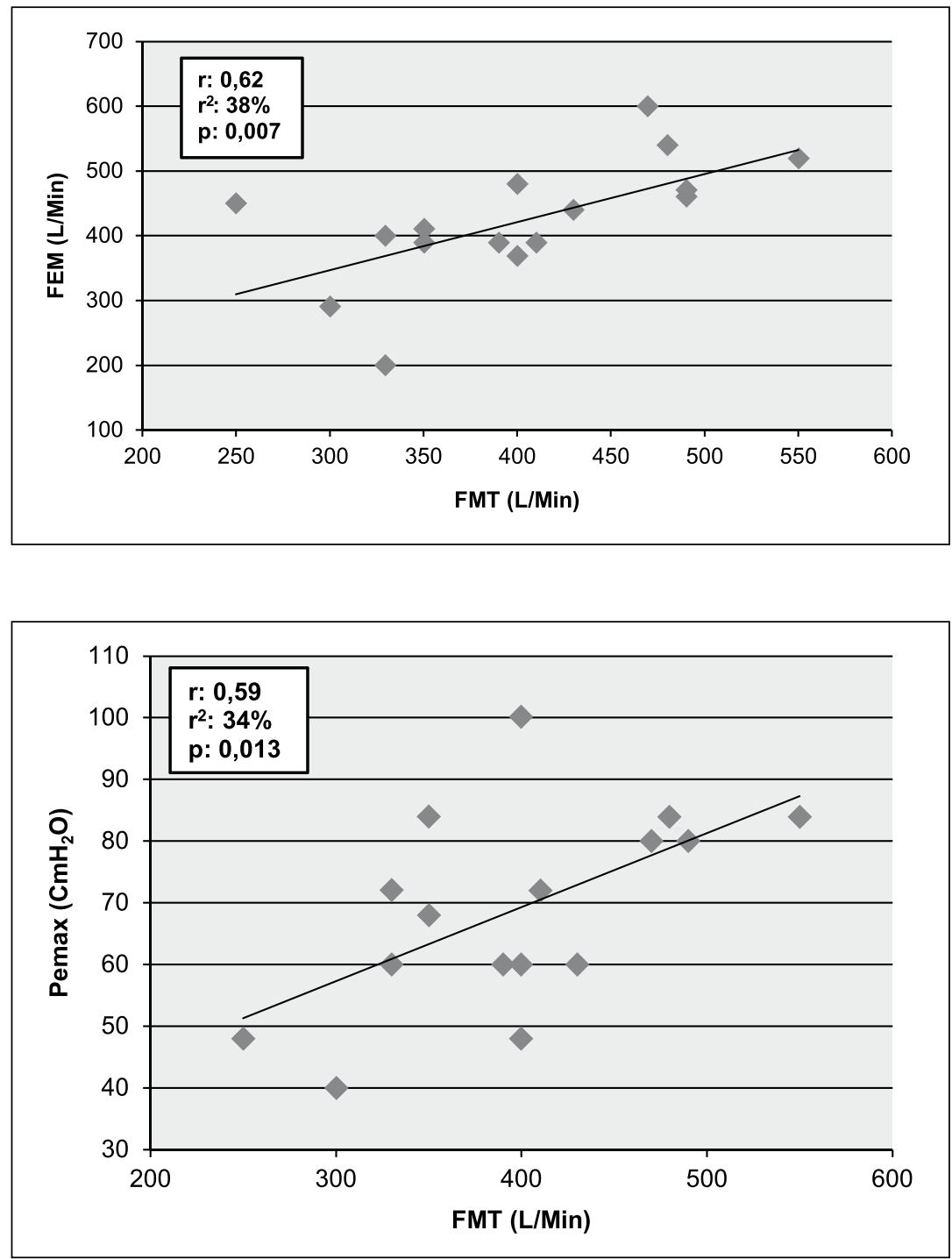

Figura 4. Correlación entre flujo espiratorio máximo y flujo máximo de tos. FEM: flujo espiratorio máximo; FMT: flujo máximo de tos.
Figura 5. Correlación entre presión espiratoria máxima y flujo máximo de tos. Pemax: presión espiratoria máxima; FMT: flujo máximo de tos. 
Esto, con el propósito de aportar antecedentes que contribuyan al diseño de futuros estudios con mayor calidad metodológica en esta línea, y aplicada a niños con patologías que cursen con debilidad de los músculos respiratorios. Los principales hallazgos fueron:

- Cada una de las variables evaluadas (Pemax, Pimax, FEM, FMT y fuerza de músculos abdominales) experimentaron incrementos significativos luego de 8 semanas de entrenamiento de fuerza abdominal.

- No se observó correlación entre el rendimiento del sit-up test y las variables de función respiratoria. Solo se observó correlación moderada entre FMT con FEM y Pemax.

Los músculos espiratorios cumplen un papel clave durante el mecanismo de la tos. Durante la fase inspiratoria se elongan optimizando la relación longitud-tensión, lo que permite la generación de mayor presión positiva intratorácica; y durante la fase compresiva se contraen contra una glotis cerrada lo que promueve la contracción isométrica de los músculo espiratorios. Este fenómeno permite que los músculos espiratorios mantengan una relación longitud-fuerza favorable para generar mayor presión positiva intraabdominal e intratorácica que puede alcanzar los $300 \mathrm{mmHg}$. Posteriormente en la fase expulsiva la glotis se abre y la presión generada en la fase compresiva permite la aceleración del flujo espiratorio alcanzando flujos de hasta $12 \mathrm{~L} / \mathrm{s}^{1}$.

Los músculos abdominales constituyen los motores primarios de la espiración activa, dentro de los cuales el recto anterior del abdomen es uno de los más importantes, activándose tanto durante el ejercicio como durante el mecanismo de la tos $^{1}$. Son escasos los reportes que han mostrado el impacto de un protocolo de fortalecimiento de músculos abdominales sobre la función respiratoria. La evidencia existente se remite al empleo de métodos pasivos de fortalecimiento, donde se ha observado que protocolos de electro-estimulación de músculos abdominales poseen impacto positivo sobre el FMT, FEM, volumen espiratorio forzado en el primer segundo, capacidad vital forzada, presión gástrica y presión esofágica en pacientes con lesión medular alta, evidenciándose aumentos significativos en las presiones y flujos generados durante las maniobras de espiración forzada y tos voluntaria asociada a la electroestimulación ${ }^{13,14}$.

En nuestro estudio, realizamos un protocolo de fortalecimiento activo de músculos abdominales constituido por ejercicios localizados dirigidos a mejorar la fuerza de dicho grupo muscular, lo que permitiría mejorar el mecanismo de la tos. En este contexto, es posible considerar que, en un sistema respiratorio normal, la capacidad generadora de flujo dependerá de la fuerza y velocidad de acortamiento muscular abdominal ${ }^{1}$, lo que podría estar reflejado en el número de abdominales que el sujeto puede realizar en un tiempo determinado. A pesar que fue posible demostrar los incrementos del FEM y FMT posterior al protocolo de entrenamiento, no observamos correlación entre FMA y las variables FEM y FMT antes de iniciar el protocolo. No obstante, se observó correlación entre la Pemax y FMT lo que demuestra la asociación existente entre la capacidad generadora de flujo y la fuerza de la musculatura espiratoria.

Respecto al entrenamiento, este se llevó a cabo dentro del ambiente educacional a través de una metodología que integra la ejecución grupal de cada uno de los ejercicios. En este contexto, la falta de supervisión personalizada en la ejecución del protocolo pudo haber afectado su cumplimiento, lo cual, pudiese ser una fuente de sesgo en el estudio. No obstante, el haberlo desarrollado en el ambiente social al que los niños y adolescentes se encuentran expuestos, incrementa la validez externa de estos resultados.

Proponemos que futuros estudios en esta línea debiesen incorporar ciertos elementos metodológicos tales como: grupo control, asignación aleatoria, método probabilístico de muestreo y enmascaramiento. Puesto que con el diseño empleado en este estudio, es imposible descartar ciertas fuentes de error sistemático como es el sesgo de medición, confusión y variabilidad biológica de la muestra.

\section{Conclusión}

Es posible concluir que, en la muestra estudiada, se observó un incremento en el nivel de fuerza muscular respiratoria, flujo espiratorio máximo y flujo máximo de tos posterior a un protocolo de entrenamiento de fuerza abdominal. No se confirma la asociación entre la fuerza de músculos abdominales y flujos espiratorios forzados, sin embargo, el flujo espiratorio máximo muestra correlación con la presión espiratoria máxima lo que sugiere la existencia de interdependencia entre la generación de flujos espiratorios forzados y la fuerza de los músculos espiratorios.

Futuros estudios son necesarios para evaluar con un mayor nivel de certeza la asociación entre el entrenamiento abdominal y variables de función respiratoria, así como también, los alcances 
clínicos que tendría la implementación de este tipo de protocolos de entrenamiento a pacientes con enfermedades cardiorrespiratorias crónicas.

\section{Bibliografía}

1.- MCCOOL F D. Global physiology and pathophysiology of cough: ACCP evidence-based clinical practice guidelines. Chest 2006; 129 (1 Suppl): 48S-53S.

2.- PARK J H, KANG S W, LEE S C, CHOI W A, KIM D H. How respiratory muscle strength correlates with cough capacity in patients with respiratory muscle weakness. Yonsei Med J 2010; 51: 392-7.

3.- KANG S W, KANG Y S, SOHN H S, PARK J H, MOON J H. Respiratory muscle strength and cough capacity in patients with Duchenne muscular dystrophy. Yonsei Med J 2006; 47: 184-90.

4.- DASSIOS T G, KATELARI A, DOUDOUNAKIS S, DIMITRIOU G. Chronic Pseudomonas aeruginosa Infection and Respiratory Muscle Impairment in Cystic Fibrosis. Respir Care 2014; 59: 363-70.

5.- DIVANGAHI M, BALGHI H, DANIALOU G, COMTOIS AS, DEMOULE A, ERNEST S, et al. Lack of CFTR in skeletal muscle predisposes to muscle wasting and diaphragm muscle pump failure in cystic fibrosis mice. PLoS Genet 2009; 5 (7): e1000586.

6.- DASSIOS T, KATELARI A, DOUDOUNAKIS S, MANTAGOS S, DIMITRIOU G. Respiratory Muscle Function in Patients With Cystic Fibrosis. Pediatr Pulmonol 2013; 48: 865-73.

7.- BACH J R. Mechanical insufflation-exsufflation. Comparison of peak expiratory flows with manually assisted and unassisted coughing techniques. Chest 1993; 104: 1553-62.

8.- FAUROUX B, GUILLEMOT N, AUBERTIN G, NATHAN N, LABIT A, CLÉMENT A, et al. Physiologic benefits of mechanical insufflation-exsufflation in children with neuromuscular diseases. Chest 2008; 133: 161-8.

9.- ILLI S K, HELD U, FRANK I, SPENGLER C M.
Effect of respiratory muscle training on exercise performance in healthy individuals: a systematic review and meta-analysis. Sports Med 2012; 42: 707-24.

10.- CHIARA T, MARTIN A D, DAVENPORT P W, BOLSER D C. Expiratory muscle strength training in persons with multiple sclerosis having mild to moderate disability: effect on maximal expiratory pressure, pulmonary function, and maximal voluntary cough. Arch Phys Med Rehabil 2006; 87: 468-73.

11.- BERLOWITZ D, TAMPLIN J. Respiratory muscle training for cervical spinal cord injury. Cochrane Database Syst Rev 2013; 7: CD008507.

12.- ZENTENO D, PUPPO H, VERA R, TORRES R, CHUN-YANG K, SALINAS P, et al. Guías de rehabilitación para niños con enfermedades respiratorias crónicas. Neumol Pediatr 2008; 3 (Supl 1): 25-33.

13.- MCBAIN R A, BOSWELL-RUYS C L, LEE B B, GANDEVIA S C, BUTLER J E. Abdominal Muscle Training Can Enhance Cough After Spinal Cord Injury. Neurorehabil Neural Repair 2013; 27: 834-43

14.- BUTLER J E, LIM J, GORMAN R B, BOSWELLRUYS C, SABOISKY J P, LEE B B, et al. Posterolateral surface electrical stimulation of abdominal expiratory muscles to enhance cough in spinal cord injury. Neurorehabil Neural Repair 2011; 25: 158-67.

15.- CÉSPEDES J, GUTIÉRREZ M, OYARZÚN M. Flujometría en la práctica de atención primaria. Rev Chil Enf Respir 2010; 26: 47-48.

16.- RODRÍGUEZ I, ZENTENO D, MANTEROLA C. Effects of home-based respiratory muscle training in children and adolescents with chronic lung disease. J Bras Pneumol 2014; 40: 626-33.

17.- AMERICAN THORACIC SOCIETY/EUROPEAN RESPIRATORY SOCIETY. ATS/ERS Statement on respiratory muscle testing. Am J Respir Crit Care Med 2002; 166: 518-624.

18.- ELBASAN B, TUNALI N, DUZGUN I, OZCELIK U. Effects of chest physiotherapy and aerobic exercise training on physical fitness in young children with cystic fibrosis. Ital J Pediatr 2012; 38: 2.
Correspondencia a:

Iván Rodríguez Núñez.

Facultad de Ciencias de la Salud, Universidad

San Sebastián. Lientur n ${ }^{\circ}$ 1457. Concepción, Chile.

Email: klgo.ivanronu@gmail.com 